\title{
Interleukin-1 receptor-associated kinase 4 as a potential biomarker: Overexpression predicts poor prognosis in patients with glioma
}

\author{
JIALIN WANG ${ }^{1 *}$, BINFENG LIU ${ }^{1 *}$, JIAWEI YAO ${ }^{2 *}$, ZHENDONG LIU $^{3 *}$, HONGBO WANG $^{4}$, BO ZHANG ${ }^{4}$, \\ XIAOYU LIAN $^{1}$, ZHISHUAI REN $^{1}$, LIYUN LIU $^{3}$ and YANZHENG GAO ${ }^{3}$ \\ ${ }^{1}$ Department of Orthopaedics, Zhengzhou University People's Hospital, Henan Provincial People's Hospital, Zhengzhou, \\ Henan 450001; ${ }^{2}$ Department of Neurosurgery, First Affiliated Hospital of Harbin Medical University and \\ Institute of Brain Science, Harbin Medical University, Harbin, Heilongjiang 150001; ${ }^{3}$ Department of Orthopaedics, \\ Henan Provincial People's Hospital, People's Hospital of Zhengzhou University, School of Clinical Medicine,
} Henan University; ${ }^{4}$ Department of Orthopaedics, Henan University People's Hospital, Henan Provincial People's Hospital, Zhengzhou, Henan 450001, P.R. China

Received April 3, 2020; Accepted January 12, 2021

DOI: $10.3892 / \mathrm{ol} .2021 .12516$

\begin{abstract}
The undetectable onset of glioma and the difficulty of surgery lead to a poor prognosis. Appropriate biomarkers for diagnosis and treatment need to be identified. Interleukin-1 receptor-associated kinase 4 (IRAK4) is involved in the initiation and progression of cancer. However, up until now, no report has revealed the relationship between IRAK4 and glioma. The present study aimed to examine the expression of IRAK4 in glioma, and to determine if there was a relationship between IRAK4 expression and clinical outcomes or survival prognosis. Thousands of glioma tissue samples and corresponding clinical information were obtained from various databases. Then a series of bioinformatics methods were used to reveal the role of IRAK4 in glioma. Finally, reverse transcription-quantitative PCR technology was used to verify the bioinformatics results. The study found that the expression of IRAK4 was significantly increased in glioma compared with the control brain tissue samples, and IRAK4, as an independent prognostic factor, shortened the overall survival time of patients with glioma. Gene Set Enrichment Analysis showed that IRAK4 promoted the activation of cell signalling pathways, such as NOD-like and Toll-like receptor signalling pathways. Co-expression analysis showed that the expression
\end{abstract}

Correspondence to: Dr Yanzheng Gao, Department of Orthopaedics, Henan Provincial People's Hospital, People's Hospital of Zhengzhou University, School of Clinical Medicine, Henan University, 7 Weiwu Road, Zhengzhou, Henan 450001, P.R. China

E-mail: yanzhenggaohn@163.com

*Contributed equally

Key words: interleukin-1 receptor-associated kinase 4, glioma, survival prognosis, biomarker of IRAK4 was correlated with CMTM6, MOB1A and other genes. The present study demonstrated the role of IRAK4 as an oncogene in the pathological process of glioma for the first time, and highlights the potential of IRAK4 as a biomarker for prognostic evaluation and treatment of glioma.

\section{Introduction}

Glioma is the most common tumour of the central nervous system and is one of the most aggressive types of human cancer (1). A study in Germany in 2011 showed that the median prognosis of survival for patients with glioma is 20 to 36 months (2). Despite advancements in glioma treatments, such as surgical resection, radiation therapy, chemotherapy and immunotherapy, there has been little recent improvement in survival rates (3). As such, a deeper understanding of glioma pathogenesis and the discovery of novel molecular biomarkers for glioma is still paramount, which will help improve the survival rate of patients with glioma.

A large number of recent studies have shown that interleukin 1 receptor-associated kinase 4 (IRAK4) may be an oncogene that plays a key role in promoting proliferation and invasion in numerous types of cancer, including colorectal cancer and hepatocellular carcinoma (4-6). IRAK4 activates transcription factors for $\mathrm{NF}-\kappa \mathrm{B}$, and is associated with aggressive pancreatic ductal adenocarcinoma that has a poorer prognosis (7). Cheng et al (6) demonstrated that IRAK4 can regulate the stemness and drug resistance of hepatocellular carcinoma cells, and is associated with tumour malignancy and poor patient survival. However, a role for IRAK4 in glioma has not been previously reported, to the best of our knowledge. Considering the role of IRAK4 in other cancer types, it was hypothesised that IRAK4 may also play an important role in glioma, and may be a potential biomarker related to the diagnosis and treatment of glioma.

The present study investigated the role of IRAK4 in glioma based on thousands of glioma samples and related 
clinical information. First of all, combined with reverse transcription-quantitative (RT-q)PCR technology and biological information analysis, the expression levels of IRAK4 in glioma samples and corresponding normal control samples were compared. Subsequently, the relationship between high expression of IRAK4 and prognosis and a series of clinical features was explored. Finally, the Gene Set Enrichment Analysis (GSEA) method was used to explain the regulatory mechanism of IRAK4. All in all, the current study aimed to provide a potential biomarker for the prognostic evaluation and treatment of glioma.

\section{Materials and methods}

Source of data and tissue samples. The Chinese Glioma Genome Atlas database (CGGA, http://www.cgga.org.cn/) is a public database containing high-throughput data and clinical characteristics of a large number of glioma samples (8). Sequencing data and corresponding clinical information of 1,018 samples from patients with glioma were obtained from the CGGA database. After deleting samples with incomplete clinical information, 748 samples were retained for subsequent multiple analyses. The detailed clinical information of the sample is presented in Table SI.

The Cancer Genome Atlas database (TCGA; https://portal. gdc.cancer.gov/) is a well-known database containing gene sequencing data and clinical characteristics of various human malignant tumour samples (9). The publicly available data of 666 glioma samples and five normal brain tissue samples were obtained and used to verify the CGGA data.

The Gene Expression Profiling Interactive Analysis (GEPIA, http://gepia.cancer-pku.cn/) database contains tumour tissue samples from TCGA data and normal control samples from the GTEx database (10). Data of IRAK4 expression in a variety of tumour tissues and corresponding normal tissues was obtained using GEPIA.

The Gene Expression Omnibus (GEO, https://www. ncbi.nlm.nih.gov/geo/) is an open public data platform (11). Two GEO datasets related to glioma were obtained, namely GSE15824 (tumour=12, normal=2) (12) and GSE50161 (tumour=34, normal=13) (13). The platforms of these two GSE data sets were GPL570.

The Human Protein Atlas (HPA, https://www.proteinatlas. org/) contains research data related to a variety of human pathology and normal histological sections (14). The immunohistochemical results of glioma (sample ID: 3241) and normal brain tissue (sample ID: 2521) were screened and obtained from this database. The immunohistochemical antibody was CAB022077.

Tissue samples from five patients with glioblastoma and five patients with epilepsy were collected at Henan Provincial People's Hospital (Zhengzhou, China) from June 2019 to September 2019. Inclusion criteria included: i) Patients with glioma diagnosed histopathologically and ii) Those who underwent surgical treatment. Exclusion criteria included: i) Patients with glioma patients without histopathological diagnosis, ii) patients with spinal involvement and iii) Patients with incomplete data records. The samples were obtained from surgical resection in the operating room. The patient's clinical information was recorded in the supplementary file Table SII. The classification of clinical characteristics of glioma patients are presented in Table SIII. The expression levels of IRAK4 in glioma and non-tumour brain tissue samples were further validated using by RT-qPCR. Patient tissue samples were immediately frozen in liquid nitrogen after being obtained, then were stored at $-80^{\circ} \mathrm{C}$ until isolation of total RNA. The study protocol was approved by The Ethics Committee of the Henan Provincial People's Hospital (Zhengzhou, China) and all experiments were performed in accordance with approved guidelines of the Henan Provincial People's Hospital.

Cell culture. Human glioma cells (A172) and human-derived astrocyte (A735) were purchased from The Cell Bank of Type Culture Collection of The Chinese Academy of Sciences. All cells were grown in incubators at $37^{\circ} \mathrm{C}$ and $5 \%$ carbon dioxide and cultured using DMEM medium plus 10\% FBS (both Thermo Fisher Scientific, Inc.).

Cell transfection. The small interfering RNA (siRNA) that specifically targeted IRAK4 was purchased from Shanghai GenePharma Co., Ltd. The RNA oligo sequence of siRNAs used in the study is presented in Table I. In total, $0.5-2 \times 10^{5}$ cells were seeding in $500 \mu 1$ of complete medium. Cell transfection was performed when the cell confluence was $50-60 \%$. Complete medium was removed and replaced with serum-free medium. Cells were transfected using Lipofectamine ${ }^{\circledR} 2000$ reagent (Invitrogen; Thermo Fisher Scientific, Inc.) and $4 \mu \mathrm{g} / \mathrm{ml}$ siRNA. The same concentration of si-negative dissolved in transfection reagent was used as thenegative control group. The same volume of transfection reagent was used as a blank control group. Cells were cultured at $37^{\circ} \mathrm{C}$ and $5 \% \mathrm{CO}_{2}$ for $6 \mathrm{~h}$ following cell transfection. Then serum-free medium was switched to complete medium. A series of follow-up experiments were performed after the cells were cultured for $48 \mathrm{~h}$. The transfection efficiency was determined using RT-qPCR at $48 \mathrm{~h}$ after transfection. The si-RNA with the greatest efficiency was then selected for further experiments.

$R T-q P C R$. Total RNA was isolated from sample tissues and corresponding cell lines after cell transfection using TRIzol ${ }^{\circledR}$ (Invitrogen; Thermo Fisher Scientific, Inc.) extraction. RNA quality and quantity were estimated by measuring absorbance at 260 and $280 \mathrm{~nm}$ using a NanoDrop One spectrophotometer (Thermo Fisher Scientific, Inc.). cDNA was synthesised from isolated total RNA by using a Transcriptor First Stand cDNA Synthesis kit (Roche Diagnostics) according to the manufacturer's instructions. A FastStart Universal SYBR Green Master (ROX) kit (Roche Diagnostics) was used for qPCR. The thermal cycling conditions were as follows: Initial denaturation at $95^{\circ} \mathrm{C}$ for $10 \mathrm{~min}$, denaturation at $95^{\circ} \mathrm{C}$ for $10 \mathrm{sec}$, annealing and extension at $60^{\circ} \mathrm{C}$ for $30 \mathrm{sec}$, for a total of 40 cycles. The RNA expression for GAPDH was used as an internal control. The primer sequences of all genes used in the study are recorded in Table II. Triplicates of each tissue sample were used for RT-qPCR. Expression of IRAK4 was calculated using the $2^{-\triangle \Delta C q}$ method and GAPDH was used as the reference gene (15). Wilcoxon rank sum test, one-way ANOVA following by Bonferroni's correction and unpaired 
Table I. RNA oligo sequence of siRNA.

\begin{tabular}{lc} 
Gene & \multicolumn{1}{c}{ RNA oligo sequence, 5'-3' } \\
\hline GAPDH-F & CAAGGTCATCCATGACAACTTTG \\
GAPDH-R & GTCCACCACCCTGTTGCTGTAG \\
IRAK4-si-1-F & CCUCAAUGUUGGACUAAUUTT \\
IRAK4-si-1-R & AAUUAGUCCAACAUUGAGGTT \\
IRAK4-si-2-F & CCAUUUCUGUUGGUGGUAATT \\
IRAK4-si-2-R & UUACCACCAACAGAAAUGGTT \\
IRAK4-si-3-F & CCACUUCAGUUGAAGCUAUTT \\
IRAK4-si-3-R & AUAGCUUCAACUGAAGUGGTT \\
IRAK4-si-NC-F & UUCUCCGAACGUGUCACGUTT \\
IRAK4-si-NC-R & ACGUGACACGUUCGGAGAATT
\end{tabular}

IRAK4, interleukin-1 receptor-associated kinase 4; si, small interfering; NC, negative control; F, forward; R, reverse.

t-tests were used for statistical analysis and the level of significance was set at $\mathrm{P}<0.05$.

GSEA. GSEA is a widely used bioinformatics analysis tool for gene enrichment analysis of gene function annotation $(16,17)$. Data from high-throughput sequencing contains the expression of a large number of genes, which can be used to investigate the relationships between genes $(18,19)$. GSEA formulates a special algorithm based on the existing research status of genes and cell signalling pathways, which can be used to explore the cell signalling pathways that a single gene may participate in the regulation $(16,17)$. The mRNA sequencing data from CGGA was used for GSEA analysis. First, batch calibration and normalization were performed using the SVA and limma packages of R software (version 3.6.1) (20). Then the RNA sequencing data was divided into high expression and low expression groups according to the expression of IRAK4. GSEA (version 4.0.3) jar software was used for enrichment analysis. The number of permutations was set to 1,000 and Kyoto Encyclopaedia of Genes and Genomes (KEGG) was set as the gene database. The statistical test standard was set as $\mathrm{P}<0.05$ and false discovery rate $(\mathrm{FDR})<0.25$.

Co-expression analysis and drug correlation analysis. The co-expression analysis on IRAK4 was performed using Pearson's correlation analysis (21). According to the P-value and the correlation coefficient value, five genes with positive or negative correlation with IRAK4 expression were selected. A heat map and circle map were constructed based these analysis results.

Statistical analysis. R software (version 3.6.1) was used for all data analysis (20). The Wilcoxon rank sum or Kruskal-Wallis tests were used to determine if there was an association between clinical characteristics and expression of IRAK4 in patients with glioma. According to the World Health Organisation (WHO) 2007 grading standard, the data from the CGGA database were classified into 'All grades of gliomas', 'WHO Grade II', 'WHO Grade III' and 'WHO Grade IV' (1). After that, survival analysis was performed for each group.
Table II. Primer sequences of reverse transcription quantitative-PCR used to verify the co-expression analysis.

\begin{tabular}{ll}
\hline Gene & \multicolumn{1}{c}{ Primer sequence, 5'-3' } \\
\hline GAPDH-F & CAAGGTCATCCATGACAACTTTG \\
GAPDH-R & GTCCACCACCCTGTTGCTGTAG \\
IRAK4-F & TCATAGGCGGCAGGAACTTA \\
IRAK4-R & ACCCAAACACTTCCCATCAG \\
CMTM6-F & TGGAGAACGGAGCGGTGTA \\
CMTM6-R & AGCCGGCCCATGAAAAAGTA \\
MOB1A-F & CAGCAGCCGCTCTTCTAAAAC \\
MOB1A-R & CCTCAGGCAACATAACAGCTTG \\
MFSD1-F & GTTGTCACCACTTTCCCCTCT \\
MFSD1-R & CAGTAACAGCACCAAAAGCCG \\
CD164-F & TCAAGTGGGGAACACGACAG \\
CD164-R & TTCGCACAGGTTGTGAGGTT \\
CMTR2-F & TTGCGGGAGCTTCATACAGG \\
CMTR2-R & CAGGTCCTCAGGGGATCAGA \\
HIST3H2BB-F & TGCCAGACCCGTCCAAAT \\
HIST3H2BB-R & TCTTCTGTGCCTTGGTGACA \\
RPPH1-F & TCCTGTCACTCCACTCCCAT \\
RPPH1-R & TGGCCCTAGTCTCAGACCTT \\
TERC-F & CGCCTTCCACCGTTCATTCTA \\
TERC-R & TGACAGAGCCCAACTCTTCG \\
HIST1H4C-F & GCAAAGGCGGAAAAGGCTTG \\
HIST1H4C-R & TAGCCGGTTTGTAATGCCCT \\
RIMS1-F & TGGAAGTCATTAGAGCACGAAGC \\
RIMS1-R & CCCAGACAATCACCTGAAGAACT \\
\hline
\end{tabular}

IRAK4, interleukin-1 receptor-associated kinase 4; F, forward; $\mathrm{R}$, reverse.

Kaplan-Meier and Cox regression survival analysis were used in the three groups 'All grades of gliomas', 'WHO Grade II' and 'WHO Grade III'. The Renyi test was used in the 'WHO Grade IV' group using SAS software (version 9.4; SAS Institute, Inc.). Univariate and multivariate Cox analyses were used to compare the effects of IRAK4 expression and other clinical features on the survival of patients with glioma. Pearson's correlation analysis was used to evaluate the degree of correlation between the expression of different genes. The Wilcoxon rank sum test was used to compare the expression of IRAK4 in the glioma and non-cancer control groups. $\mathrm{P}<0.05$ was considered to indicate a statistically significant difference.

\section{Results}

Clinical features and the association between IRAK4 expression and clinical features in patients with glioma. The glioma sample data obtained from the CGGA database was screened and normalized. Its clinical characteristics are presented in Table SI. The patients were divided into different groups according to various clinical characteristics, and the expression levels of IRAK4 in each group were compared. Fig. 1 shows that the expression level of IRAK4 in patients 
A

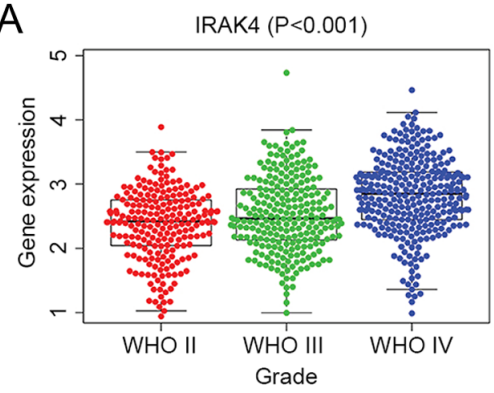

B

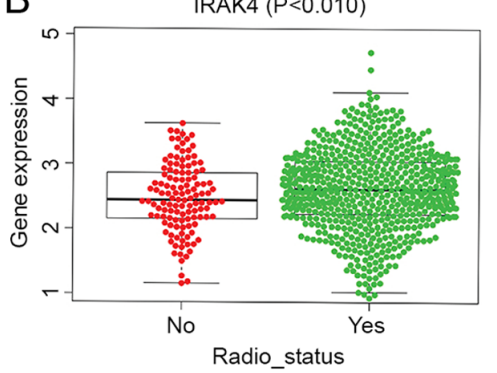

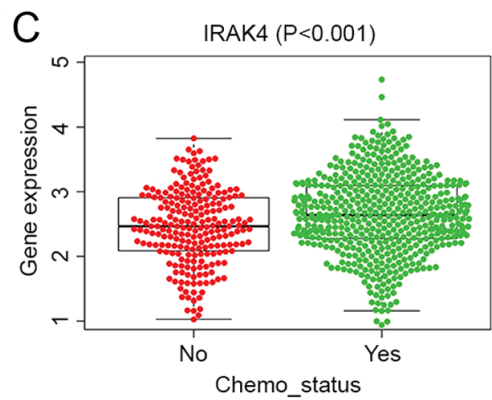
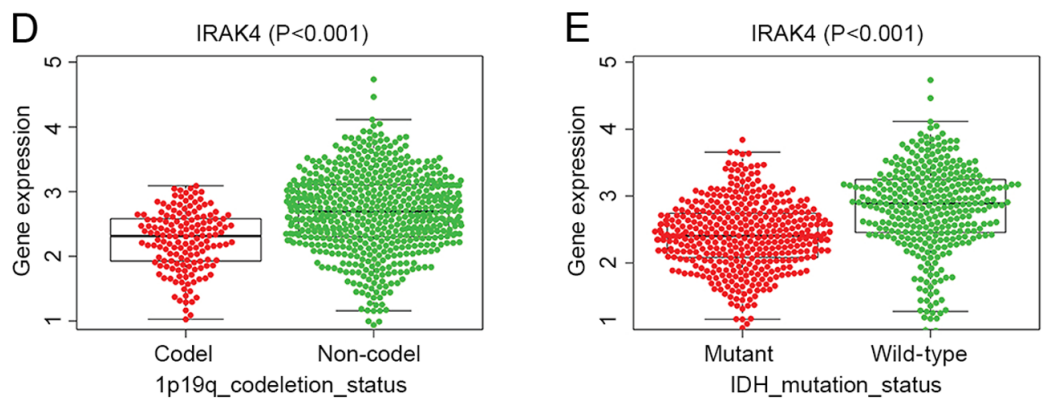

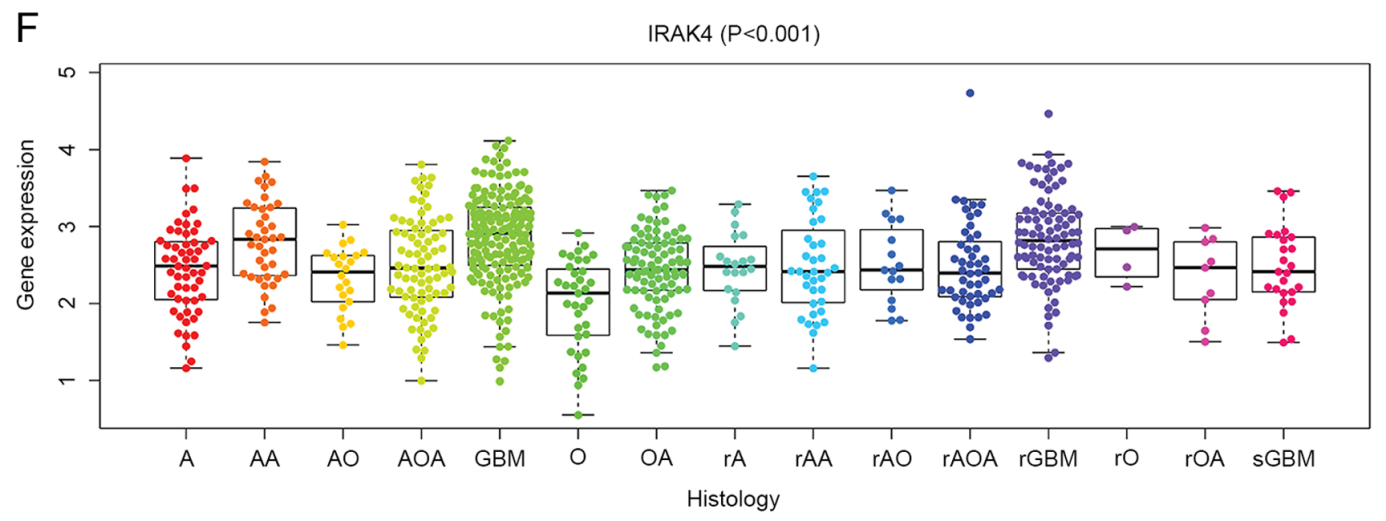

Figure 1. Association between IRAK4 expression and clinicopathological features in glioma. (A) Grade, (B) radiotherapy status, (C) chemotherapy status, (D) $1 \mathrm{p} 19 \mathrm{q}$ co-deletion status, (E) IDH mutation status and (F) histology. IRAK4, interleukin-1 receptor-associated kinase 4.

with glioma from CGGA may be associated with a variety of clinical and molecular characteristics. The expression of IRAK4 was significantly higher in patients with higher WHO grade, isocitrate dehydrogenase (IDH) wild-type and 1p19p non-co-deletion compared with in patients with lower WHO Grade, IDH mutation and $1 \mathrm{p} 19 \mathrm{p}$ co-deletion (all $\mathrm{P}<0.001$; Fig. 1A, D and E).

High expression of IRAK4 can lead to a poor prognosis. According to the expression level of IRAK4, CGGA mRNA sequencing data of glioma were divided into high and low expression groups. After that, Kaplan Meier method was used to draw the overall survival curve. The results showed that high IRAK4 expression group is significantly associated with a shorter survival time of patients with glioma of all grades $(\mathrm{P}<0.001)$, WHO-II grade gliomas $(\mathrm{P}=0.011)$ and WHO-III grade gliomas $(\mathrm{P}<0.001)$ (Fig. 2A-C). Receiver operating curve (ROC) was used to explore whether the expression level of IRAK4 had a certain prognostic value in glioma. The results showed that the expression level of IRAK4 had a certain predictive value for the 5-year survival rate (area under the curve $>0.7$ ) in Fig. 3.
Univariate analysis and multivariate analysis were used to determine whether the high expression of IRAK4 is an independent risk factor leading to poor prognosis in patients with glioma. It must be emphasized that the high expression of IRAK4, Primary Recurrent Secondary type, higher WHO grade and age can all be used as independent risk factors and lead to poor prognosis $[\mathrm{P}<0.05$, hazard ratio $(\mathrm{HR})>1]$. Meanwhile, postoperative chemotherapy, IDH mutation status and $1 \mathrm{p} 19 \mathrm{q}$ co-deletion status were independent protective factors that lead to an improved prognosis $(\mathrm{P}<0.05, \mathrm{HR}<1)$ in Fig. 4.

Validation of the association between IRAK4 and glioma. After performing RT-qPCR on clinical samples, Fig. 5A shows that the expression level of IRAK4 in glioma tissues $(\mathrm{n}=5)$ was increased $(\mathrm{P}<0.001)$ compared with normal brain tissues $(n=5)$. The results showed that the expression level of IRAK4 was higher in glioma tissues compared with that in normal tissues $(\mathrm{P}=0.003$; Fig. $5 \mathrm{C})$. The overall survival of patients in the IRAK4 high expression group was significantly lower compared with that of the IRAK4 high expression group, which suggested that the abnormally high expression 
A

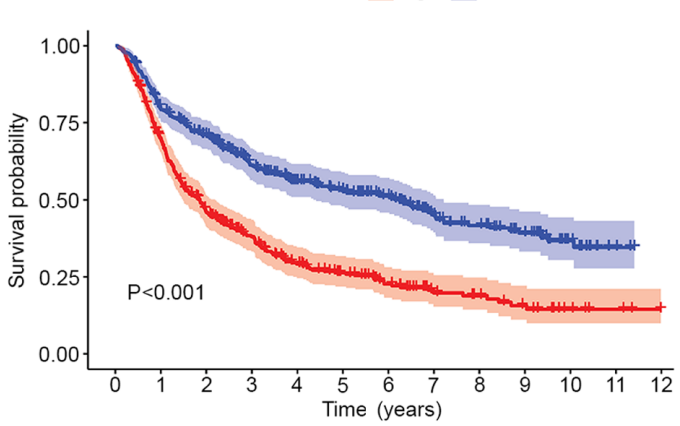

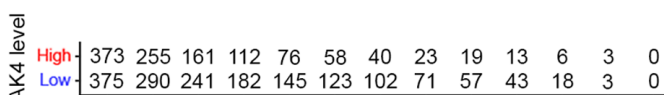

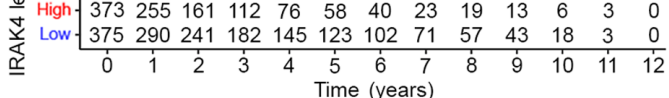

C

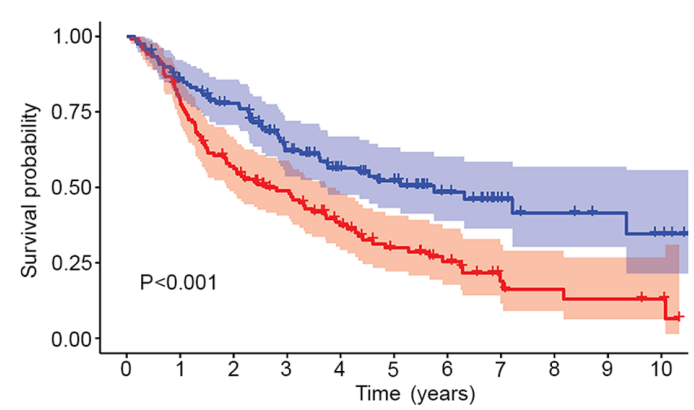

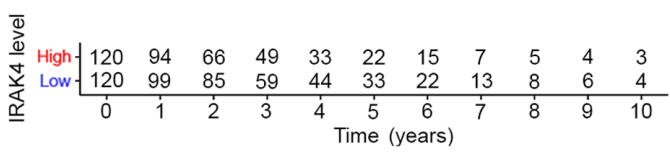

B IRAK4 level + High + Low

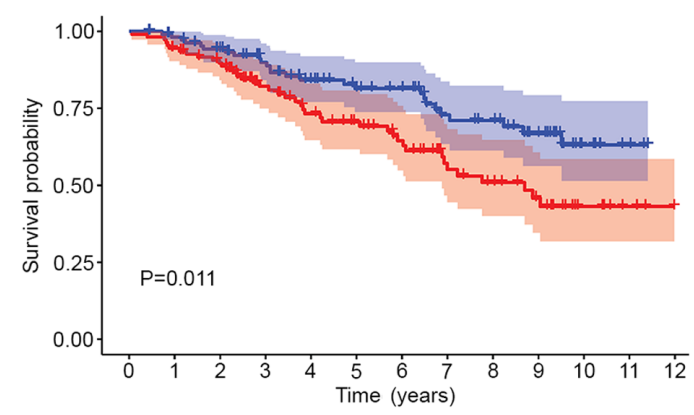

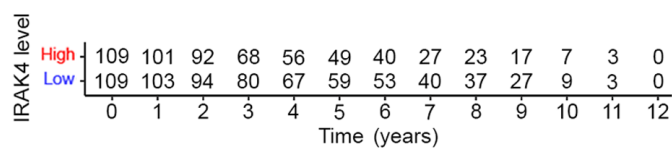

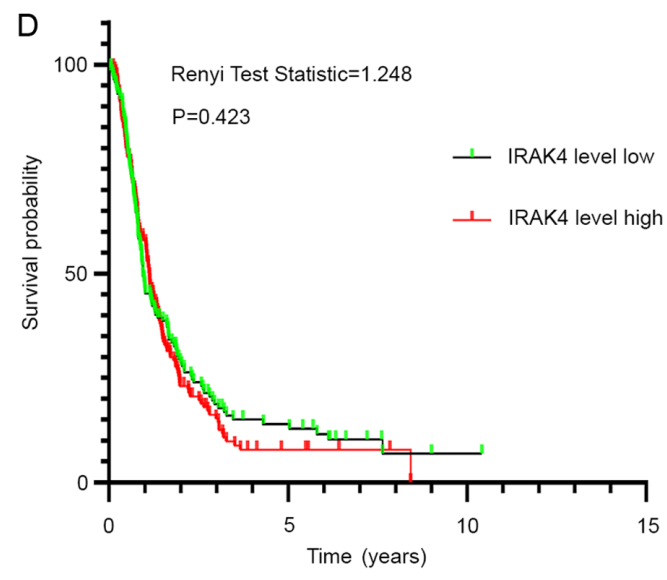

Figure 2. Association between expression of interleukin-1 receptor-associated kinase 4 and overall survival in the Chinese Glioma Genome Atlas database glioma patient cohort and its corresponding subgroups. (A) All grades of gliomas, World Health Organisation grades (B) II, (C) III and (D) IV.

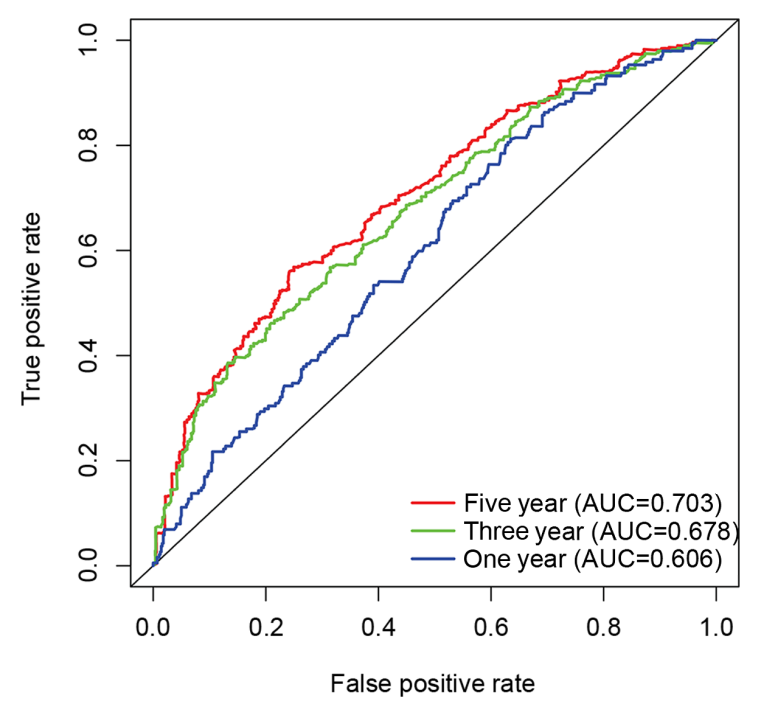

Figure 3. Assessment of interleukin-1 receptor-associated kinase 4 expression as a predictor of 1-, 3- and 5-year survival of patients with glioma. AUC, area under the curve.

of IRAK4 was related to poor prognosis ( $\mathrm{P}<0.001$; Fig. $5 \mathrm{~B})$. The analysis of the GEPIA database showed that the expression level of IRAK4 in GBM and LGG samples was significantly higher compared with that in the corresponding normal control samples ( $\mathrm{P}<0.05$; Fig. $5 \mathrm{D}$ and $\mathrm{E})$. The analysis of GSE15824 showed that the expression level of IRAK4 in glioma tissue samples was significantly higher compared with that of normal brain tissue $(\mathrm{P}=0.044$; Fig. $5 \mathrm{~F})$. The analysis of GSE50161 showed that the expression level of IRAK4 in glioma tissue samples was significantly higher compared that of normal brain tissue $(\mathrm{P}<0.001$; Fig. $5 \mathrm{G})$. In addition to detection at the mRNA level, the protein expression level of IRAK4 was assessed using the HPA database. The results showed that the protein expression level of IRAK4 in glioma tissue was higher compared with that in normal brain tissue (Fig. 5H). In summary, Fig. 5 shows that IRAK4 had abnormally high expression relative to normal control samples at the mRNA and protein levels from multiple data sources.

GSEA analysis. GSEA was used to form a comparison between datasets from patients that showed either high or low expression of IRAK4 and identify signalling pathways that had different levels of activity in glioma. There were significant differences (nominal $\mathrm{P}<0.05$ and FDR $<0.25$ ) in enrichment of pathways identified using KEGG analysis. It was reported 


$\begin{array}{lrr}\text { A } & \text { P-value } & \text { Hazard ratio } \\ \text { IRAK4 } & <0.001 & 2.193(1.873-2.566) \\ \text { PRS_type } & <0.001 & 2.123(1.818-2.478) \\ \text { Histology } & <0.001 & 4.487(3.695-5.449) \\ \text { Grade } & <0.001 & 2.883(2.526-3.291) \\ \text { Gender } & 0.655 & 1.044(0.866-1.258) \\ \text { Age } & <0.001 & 1.624(1.345-1.960) \\ \text { Radio } & 0.571 & 0.929(0.720-1.199) \\ \text { Chemo } & <0.001 & 1.647(1.328-2.044) \\ \text { IDH_mutation } & <0.001 & 0.317(0.262-0.384) \\ \text { 1p19q_codeletion } & <0.001 & 0.231(0.169-0.315)\end{array}$

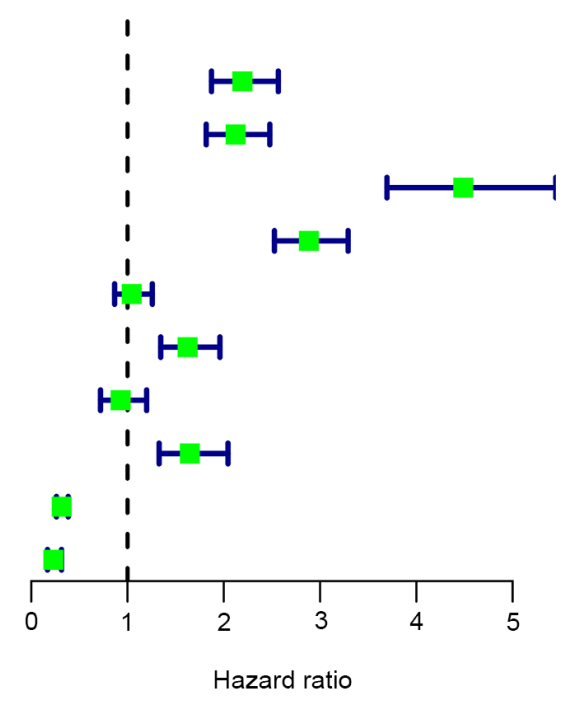

$\begin{array}{lr} & \text { P-value } \\ \text { IRAK4 } & <0.001 \\ \text { PRS_type } & <0.001 \\ \text { Histology } & 0.112 \\ \text { Grade } & <0.001 \\ \text { Gender } & 0.597 \\ \text { Age } & 0.006 \\ \text { Radio } & 0.162 \\ \text { Chemo } & <0.001 \\ \text { IDH_mutation } & 0.003 \\ \text { 1p19q_codeletion } & <0.001\end{array}$

Hazard ratio
$1.476(1.258-1.733)$
$2.023(1.718-2.382)$
$0.699(0.450-1.087)$
$2.688(1.969-3.669)$
$1.053(0.870-1.275)$
$1.323(1.082-1.618)$
$0.823(0.627-1.081)$
$0.667(0.524-0.849)$
$0.692(0.544-0.879)$
$0.418(0.299-0.584)$

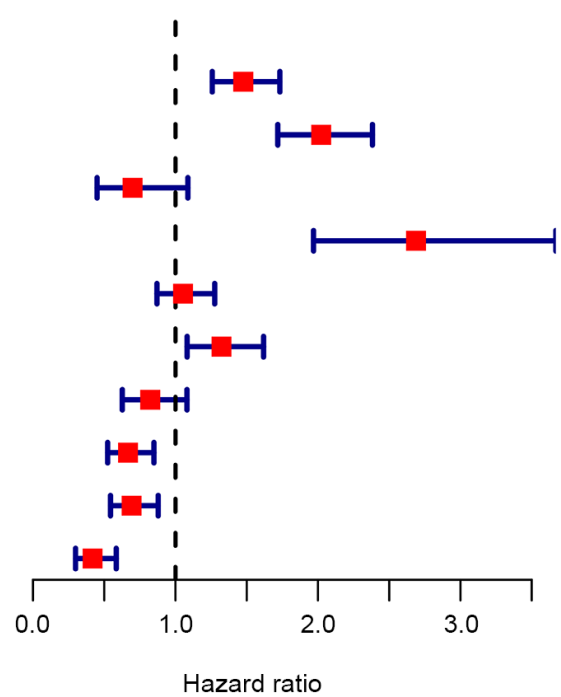

Figure 4. Abnormally high expression of IRAK4 is an independent risk factor for the prognosis of glioma. (A) Univariate regression analysis to determine correlation of IRAK4 expression with glioma patient survival in the CGGA database. (B) Multivariate regression analysis to determine correlation of IRAK4 expression with patient survival in CGGA. IRAK4, interleukin-1 receptor-associated kinase 4; CGGA, Chinese Glioma Genome Atlas; PRS, Primary Recurrent Secondary; IDH, isocitrate dehydrogenase.

that focal adhesion, JAK-STAT, VEGF, chemokine, NOD-like receptor (NLR) and Toll-like receptor (TLR) signalling pathways were differentially enriched in the high IRAK4 expression glioma phenotype (Fig. 6). These results suggested that IRAK4 may play a regulatory role in the pathological process of glioma via the aforementioned cellular signalling pathways.

Co-expression analysis of IRAK4. A heat map of co-expression representing the top 20 genes that were most significantly negatively and positively correlated with IRAK4 is presented in Fig. 7A. Co-expression networks were constructed from gene expression data relative to normal levels of expression. In the co-expression network, each node represented a gene in the network, and gaps represented two related genes (the shorter the distance between nodes, the stronger the correlation between the genes). A string connection was a close correlation between these genes and indicated that regulatory relationships may exist. With the increasing of expression level of IRAK4, there was a positive relationship with the expression level of CMTM6, MOB1A, MFSD1, CD164 and CMTR2, and a negative relationship with the expression level of HIST3H2BB, RPPH1, TERC, HIST1H4C and RIMS1 (Fig. 7B).

After the A172 glioma cell line was cultured and the expression of IRAK4 was knocked down by gene interference technology, the expression level of related genes was detected by RT-qPCR. The expression level of IRAK4 in the A172 glioma cell line was significantly higher compared with that in HA $(\mathrm{P}<0.05$; Fig. 8A). After gene interference, the expression of IRAK4 was significantly decreased in A172 cells (all P<0.0001; Fig. 8B). siRNA-3 had the highest transfection efficiency and so was selected for further experiments. After knockdown of IRAK4 in A172 cells, the expression levels of IRAK4 positive related genes, such as CMTM6 and MOB1A, were also decreased, which was consistent with the results of the co-expression analysis of this study. However, MFSD1, CD164 and CMTR2 levels were significantly 

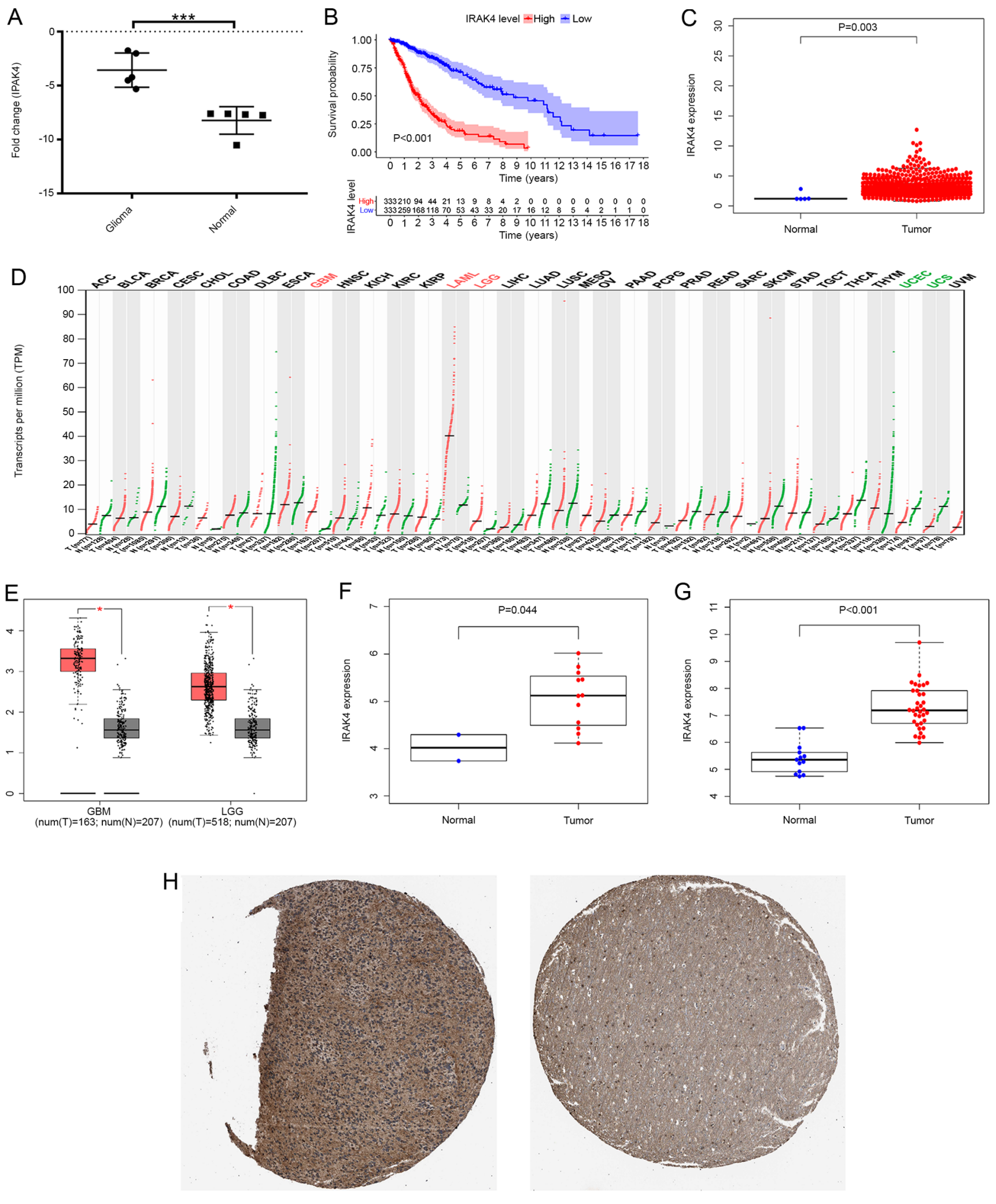

Glioma tissue

Normal brain tissue

Figure 5. Expression level of IRAK4 in glioma is higher compared with that of normal control brain tissue. (A) Reverse transcription quantitative-PCR showed that IRAK4 was highly expressed in glioma compared with non-tumour brain tissue. (B) Elevated IRAK4 expression in glioma compared with non-tumour brain tissue in TCGA glioma cohort. (C) Kaplan-Meier curve of TCGA data. (D) Expression level of IRAK4 in various tumours relative to corresponding normal tissues in the GEPIA database. Red tumour name tag indicates that IRAK4 is highly expressed in tumour tissue. Green tumour name tag indicates that IRAK4 is highly expressed in tumour tissue. Black tumour name label indicates that the expression level of IRAK4 in tumour tissue and corresponding normal tissues has no significant statistical significance. (E) Expression level of IRAK4 in GBM, LGG and their corresponding normal brain tissues in GEPIA database. (F) Expression level of IRAK4 was highly increased in glioma tissue $(n=12)$ compared with normal brain tissue (n=2) in the GSE15824 dataset. (G) Expression level of IRAK4 was highly increased in glioma tissue $(n=34)$ compared with normal brain tissue $(n=13)$ in the GSE50161 dataset. (H) Protein expression level of IRAK4 was markedly increased in glioma tissue compared with normal brain tissue by immunohistochemistry in the Human Protein Atlas database. ${ }^{*} \mathrm{P}<0.05$ and ${ }^{* * * *} \mathrm{P}<0.001$ vs. respective control. IRAK4, interleukin-1 receptor-associated kinase 4; TCGA, The Cancer Genome Atlas; GBM, glioblastoma; LGG, low-grade glioma; T, tumour; N, normal. 
A

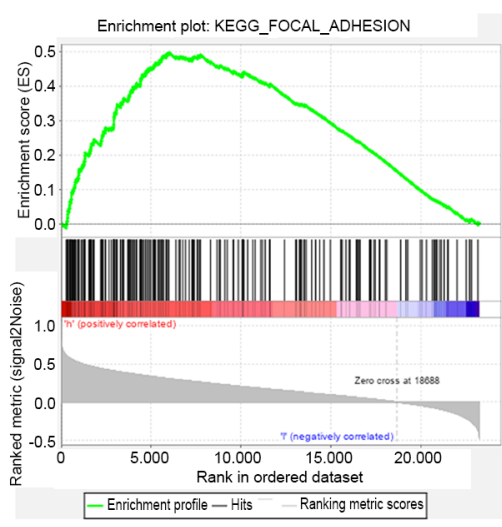

D

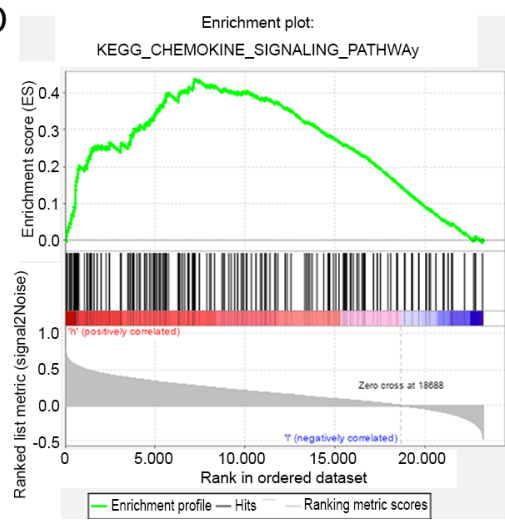

B

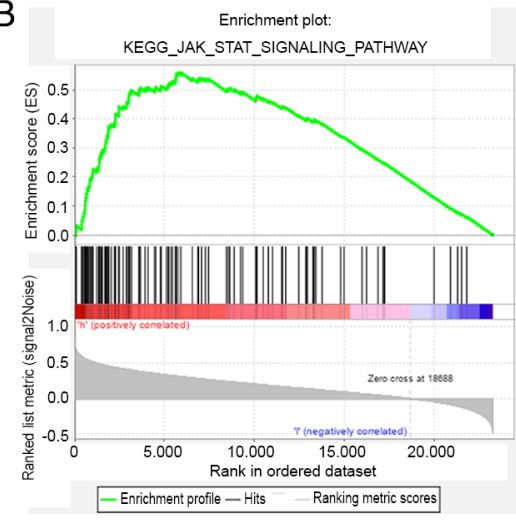

E

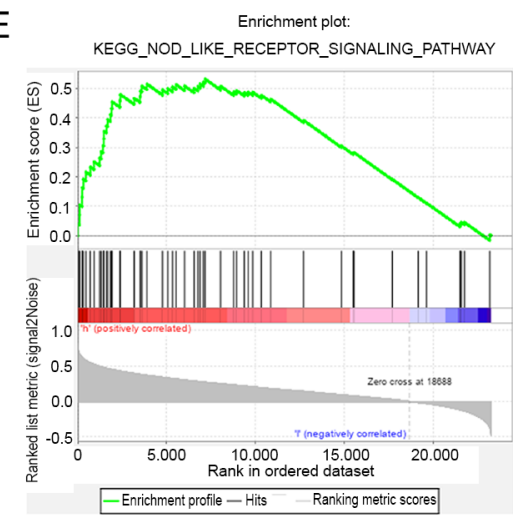

C

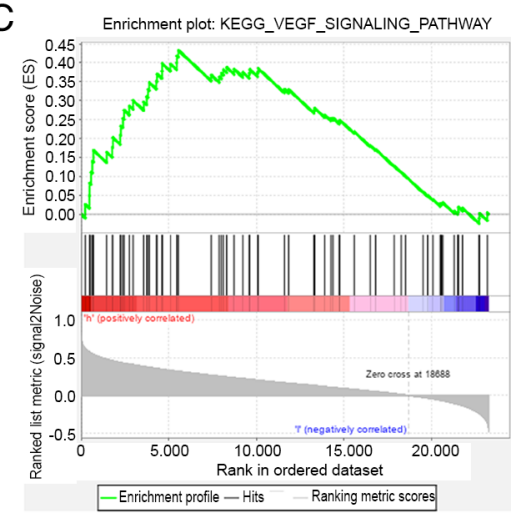

$\mathrm{F}$

F Enichment plot:

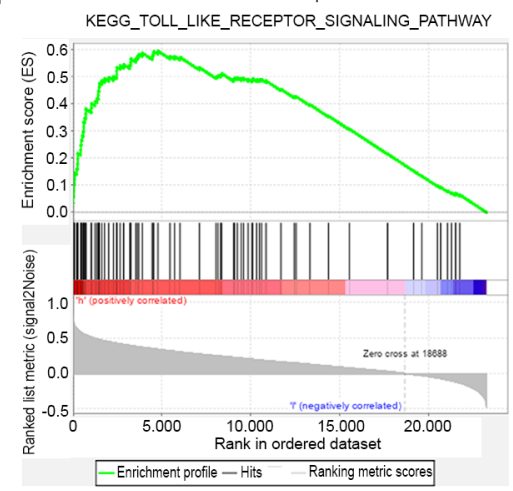

Figure 6. Enrichment plots from GSEA. GSEA identified (A) focal adhesion, (B) JAK-STAT, (C) VEGF, (D) chemokine, (E) NOD-like receptor and (F) Toll-like receptor signalling pathways as being differentially enriched in the high expression of interleukin-1 receptor-associated kinase 4 phenotype. GSEA, Gene Set Enrichment Analysis; KEGG, Kyoto Encyclopaedia of Genes and Genomes.

A

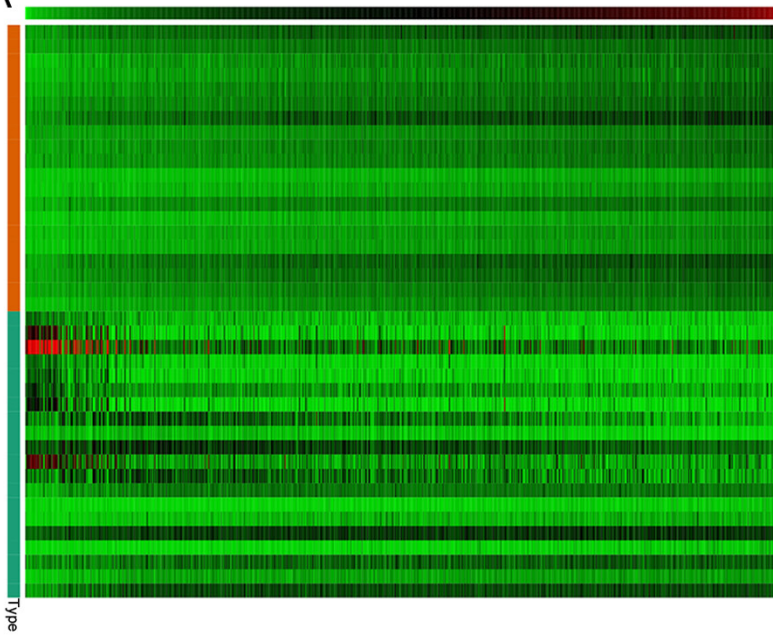

B

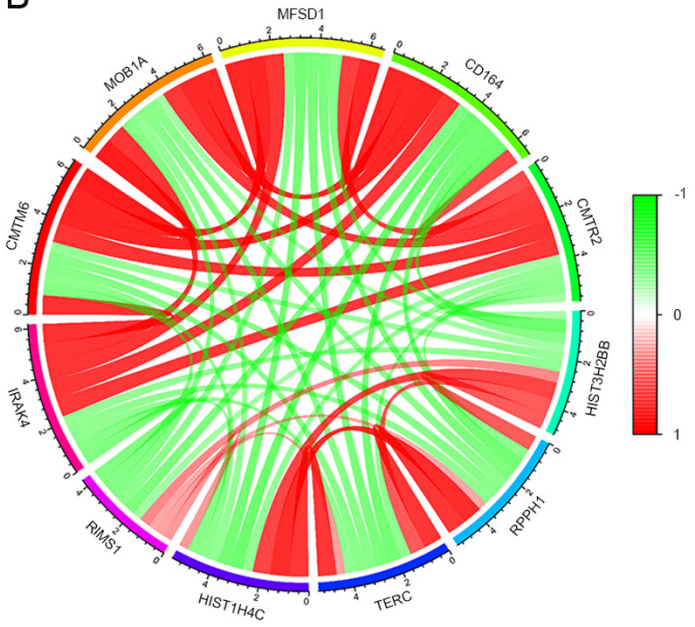

Figure 7. Co-expression analysis of IRAK4 and related drug analysis by connectivity map. (A) Heat map representing top 20 genes that are positively and negatively correlated with IRAK4 expression in patients with glioma. (B) Co-expression networks show top five genes that are positively and negatively correlated with IRAK4 expression in patients with glioma. IRAK4, interleukin-1 receptor-associated kinase 4.

increased $(\mathrm{P}<0.05, \mathrm{P}<0.001$ and $\mathrm{P}<0.01$, respectively; Fig. $8 \mathrm{C})$. After knockdown of IRAK4 in A172 cells, the expression of IRAK4 negatively related genes, such as HIST3H2BB, TERC, HIST1H4C and RIMS1, were significantly increased $(\mathrm{P}<0.0001$ or $\mathrm{P}<0.01)$, which was consistent with the results of the co-expression analysis of this study., but RPPH1 did not change significantly (Fig. 8D).

\section{Discussion}

Up to now, the etiological mechanism of glioma has not been fully elucidated. It has been reported that gene level changes have an important impact on the pathological process of glioma (22). Revealing the function of gene is helpful to clarify the pathogenesis of glioma. A large number of studies have 
A

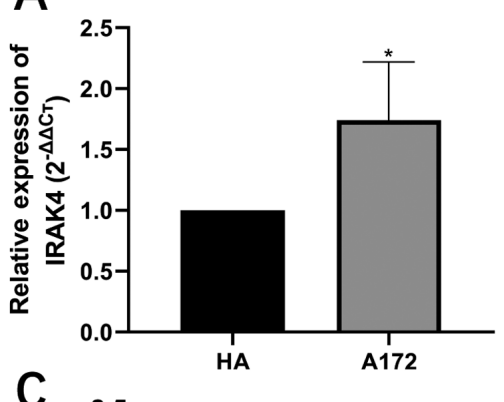

C
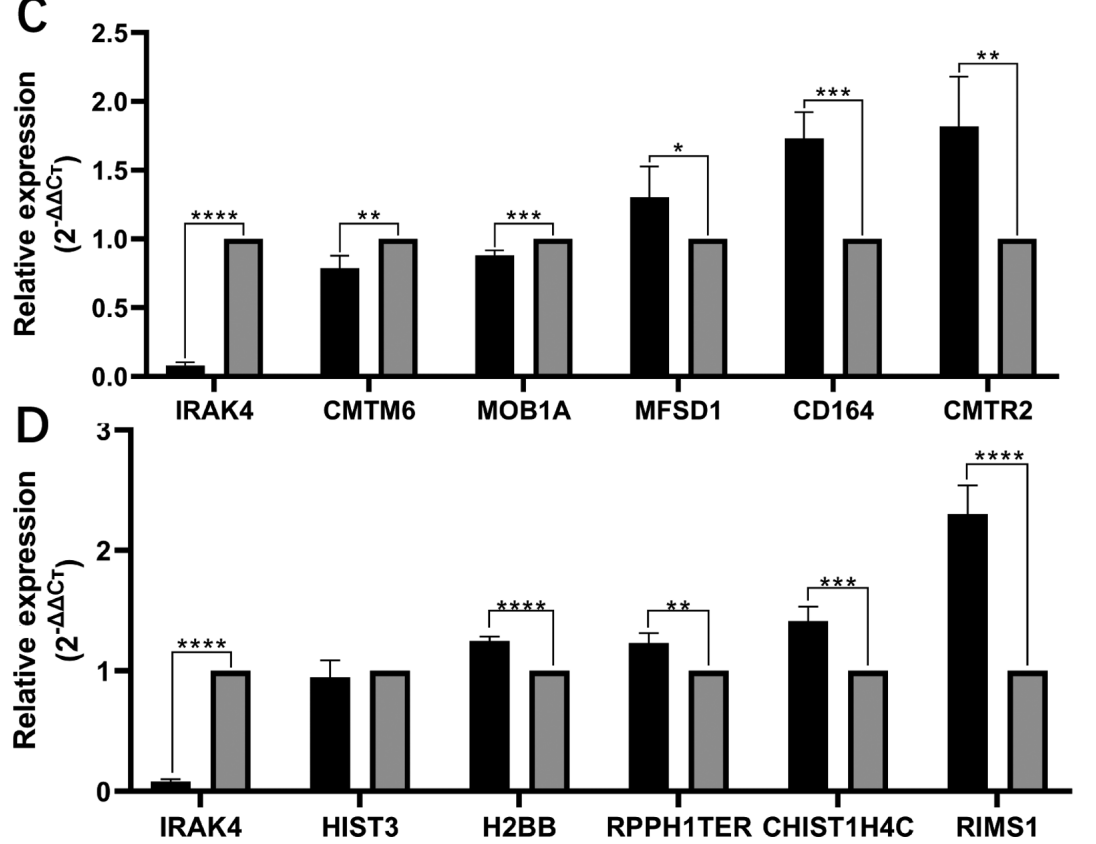

B

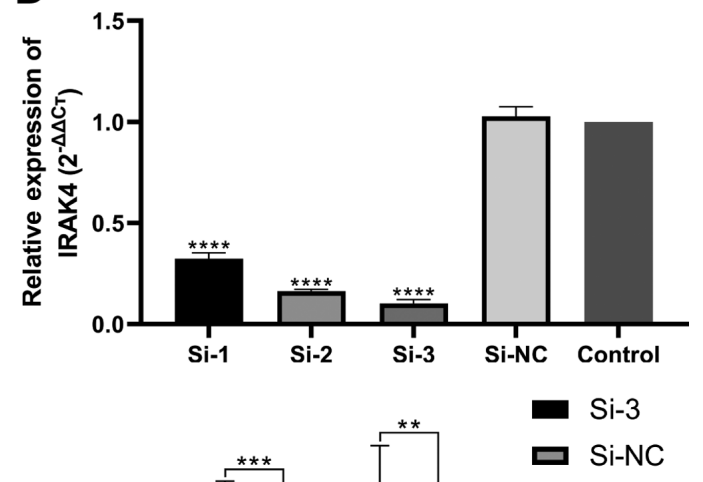

口 Si-NC

Figure 8. Expression of IRAK4 in glioma cells and the co-expression of IRAK4 by reverse transcription-quantitative PCR. (A) IRAK2 expression was increased in the glioma cell line (A172) relative to the HA line. (B) Knockout efficiency of the three IRAK4 siRNAs. (C) Expression of five genes positively correlated with IRAK4 after cell transfection and gene knockout. (D) Expression of five genes negatively correlated with IRAK4 after cell transfection and gene knockout. ${ }^{*} \mathrm{P}<0.05,{ }^{* *} \mathrm{P}<0.01,{ }^{* * *} \mathrm{P}<0.001$ or ${ }^{* * * * *} \mathrm{P}<0.0001$ vs. respective control. IRAK4, interleukin-1 receptor-associated kinase 4; HA, human astrocyte; si, small interfering.

confirmed that IRAK4, as an oncogene, plays an important regulatory role in tumorigenesis (4-6), but its function in glioma has not been revealed so far (23). The present study explored the relationship between IRAK4 and glioma. The purpose of the study was to elucidate the effect of IRAK4 on the prognosis of patients with glioma and to investigate the association between IRAK4 and clinical variables.

The current study demonstrated that high expression of IRAK4 was correlated with more advanced WHO grade, IDH wild-type and $1 \mathrm{p} 19 \mathrm{q}$ non-co-deletions. These three characteristics played an important role in the 2007 and 2016 updated WHO classifications $(1,5)$. The previous study showed that the higher the WHO classification, the higher the malignant degree of glioma and the worse the prognosis $(1,5)$. IDH mutations and $1 \mathrm{p} 19 \mathrm{q}$ often occur concurrently and are currently considered to be protective factors for the prognosis of glioma (24). This coincides with the present univariate and multivariate Cox analysis results. Subsequent survival analysis and ROC curves further suggested that abnormally high expression of IRAK4 could lead to poor prognosis of patients with glioma. Based on this, it was predicted that IRAK4 may be a potential biomarker or oncogene involved in the occurrence and development of glioma. Therefore, to investigate this hypothesis, the expression levels of IRAK4 mRNA and protein were investigated in multiple cell lines, tissue samples and databases (GEPIA, TCGA, GEO and HPA). The results demonstrated that the expression level of IRAK4 in glioma was higher compared with that in normal control.

The specific function of IRAK4 in glioma was still unclear, so based on the hypothesis that IRAK4 may be an oncogene, GSEA was used to predict the cell signalling pathways that IRAK4 may be involved in regulating. GSEA results showed that IRAK4 may be involved in the regulation of cell signalling pathways, such as VEGF, JAK-STAT, TLR signalling, focal adhesion, chemokine and NLR. The important role of VEGF in tumorigenesis and development makes it an important therapeutic target for cancer (25). Previous study has shown that inhibition of VEGF signalling may increase overall survival time in patients with glioma (26). Previous studies have shown that the JAK/STAT pathway plays an important role in the progression of glioma, and that activation of JAK-STAT signalling is predictive of poor prognosis in patients with glioma $(27,28)$. A previous study also demonstrated that IRAK4 modulates the response to temozolomide in glioma via the TLR and NF- $\mathrm{NB}$ signalling pathways (29). This is consistent with results of the present study in the 
correlation analysis of clinical features, which showed that increased IRAK4 is associated with patients with glioma receiving chemotherapy. CD155/poliovirus receptor enhances glioma cell invasion and migration by regulating adhesion signals and focal adhesion dynamics (30). Numerous chemokines are potential therapeutic targets for glioma including CCR7, which can activate matrix metalloproteinase 2/9 through NF- $\mathrm{KB}$ signalling to regulate invasion and migration of TGF- $\beta 1$-induced human glioma cells (31). In summary, the present GSEA predicted cell signalling pathways that IRAK4 might be involved in regulation.

It is well known that oncogenes can often be used as therapeutic targets for cancer suppression therapy (32). However, changes in the expression level of a single gene in organisms often lead to changes in the expression levels of other genes, thus playing a synergistic role in the regulation of disease progression (33). Therefore, to further explore the effect of IRAK4 on other genes, the current study further performed a co-expression analysis to identify more genes that are potentially linked to glioma. The co-expression network suggested that the expression levels of CMTM6, MOB1A, MFSD1, CD164 and CMTR2 were positively correlated with IRAK4, but negatively correlated with HIST3H2BB, RPPH1, TERC, HIST1H4C and RIMS. To improve the reliability of the prediction results, IRAK4 was knocked down in the A172 glioma cell line, and changes in the levels of the aforementioned co-expression genes were assessed using RT-qPCR technology. The results showed that the expression level of MFSD1, CD164 and CMTR2 were increased contrary to our predicted results, while the expression level of RPPH1 did not change significantly. Other genes co-expressed with IRAK4 had increased expression levels, consistent with the expected outcome. These co-expression genes may affect the pathological process of glioma through synergistic effect with IRAK4, but the specific mechanism needs to be further studied. This conclusion is supported by previous report. For example, the overexpression of CMTM6 was associated with poor prognosis in glioma and clinical features (34).

The present study used data from public databases to fully reveal the relationship between overexpression of IRAK4 and prognosis and clinical information of patients with glioma. However, there were some limitations. Firstly, as the public database aggregates information from multiple treatment centres, there were inevitable information gaps, inconsistent data collection and processing. In the analysis process, the impact of the difference in the patient's detailed treatment plan, such as the scope of surgical resection and differences in radiotherapy and chemotherapy regimens, could not be minimised. However, because of this, multiple databases were verified and compared, which makes the results of the current study more objective and authentic. Secondly, the number of healthy controls in public databases is relatively small compared with the number of patients with glioma, and this may introduce errors in statistical analysis. Therefore, RT-qPCR was used to validate differences in IRAK4 expression levels between glioma tissue and control non-cancer brain tissue. In addition, the present research still has certain limitations. When analysing the relationship between the expression level of IRAK4 and clinical characteristics, the two clinical characteristics of radiotherapy and chemotherapy were introduced. However, specific treatment modalities of radiation or chemotherapy may render notably different results, and therefore simply categorizing by radiotherapy or chemotherapy is of poor clinical significance.

To the best of our knowledge, the present study is the first to link the overexpression of IRAK4 with the decreased survival rate of patients with glioma. The current research suggested that IRAK4 may be a potential oncogene involved in the regulation of cell signalling pathways in glioma. IRAK4 may be a novel biomarker for prognostic evaluation and treatment of glioma.

\section{Acknowledgments}

Not applicable.

\section{Funding}

This work was supported by The Thousand Talents Plan of Henan province, Henan Provincial Innovation and Outstanding Talent Program (grant no. 154200510027), The 2016 Henan Provincial Science and Technology Research Project: Construction and Clinical Application of Digital Precision Spine Surgery Technology System (grant no. 162102310018) and The 2018 Henan provincial Medical Science and Technology Tackling Program Provincial-ministerial Co-construction Project (grant no. SBGJ2018076).

\section{Availability of data and materials}

The datasets used and/or analysed during the current study are available in The Cancer Genome Atlas [http://www.cgga.org.cn/] and the Chinese Glioma Genome Atlas [http://www.cgga.org. $\mathrm{cn} / \mathrm{]} \mathrm{repositories.} \mathrm{The} \mathrm{additional} \mathrm{datasets} \mathrm{used} \mathrm{and/or} \mathrm{analysed}$ during the current study are available from the corresponding author on reasonable request.

\section{Authors' contributions}

JW, ZL, BL, LL and YG designed the study. JW conducted basic experimental verification. JY, HW, LL and YG collected the tissue samples. JW and ZL reviewed the raw data and confirmed the authenticity of all raw data. JW and BZ performed the analysis. XL and ZR collected data. ZL and BL drafted the manuscript. LL and YG gave final approval of the version to be published. All authors read and approved the final manuscript.

\section{Ethics approval and consent to participate}

The study protocol was approved by The Ethics Committee of the Henan Provincial People's Hospital (Zhengzhou, China). The use of patient samples conformed to the declaration of Helsinki. All patients provided informed written consent.

\section{Patient consent for publication}

Not applicable.

\section{Competing interests}

The authors declare that they have no competing interests. 


\section{References}

1. Louis DN, Ohgaki H, Wiestler OD, Cavenee WK, Burger PC, Jouvet A, Scheithauer BW and Kleihues P: The 2007 WHO classification of tumours of the central nervous system. Acta Neuropathol 114: 97-109, 2007.

2. Brandes AA, Tosoni A, Spagnolli F, Frezza G, Leonardi M, Calbucci F and Franceschi E: Disease progression or pseudoprogression after concomitant radiochemotherapy treatment: Pitfalls in neurooncology. Neuro Oncol 10: 361-367, 2008.

3. Ho VK, Reijneveld JC, Enting RH, Bienfait HP, Robe P, Baumert BG and Visser O; Dutch Society for Neuro-Oncology (LWNO): Changing incidence and improved survival of gliomas. Eur J Cancer 50: 2309-2318, 2014.

4. Kargbo RB: PROTAC degradation of IRAK4 for the treatment of cancer. ACS Med Chem Lett 10: 1370-1371, 2019.

5. Li Q, Chen Y, Zhang D, Grossman J, Li L, Khurana N, Jiang H, Grierson PM, Herndon J, DeNardo DG, et al: IRAK4 mediates colitis-induced tumorigenesis and chemoresistance in colorectal cancer. JCI Insight 4: e130867, 2019.

6. Cheng BY, Lau EY, Leung HW, Oi-Ning Leung C, Ho NP, Gurung S, Cheng LK, Lin CH, Cheuk-Lam Lo R, Ma S, et al: IRAK1 augments cancer stemness and drug resistance via the AP-1/AKR1B10 signaling cascade in hepatocellular carcinoma. Cancer Res 78: 2332-2342, 2018.

7. Zhang D, Li L, Jiang H, Knolhoff BL, Lockhart AC, Wang-Gillam A, DeNardo DG, Ruzinova MB and Lim KH: Constitutive IRAK4 activation underlies poor prognosis and chemoresistance in pancreatic ductal adenocarcinoma. Clin Cancer Res 23: 1748-1759, 2017.

8. Wang Z, Wang Z, Zhang C, Liu X, Li G, Liu S, Sun L, Liang J, $\mathrm{Hu} \mathrm{H}$, Liu Y, et al: Genetic and clinical characterization of B7-H3 (CD276) expression and epigenetic regulation in diffuse brain glioma. Cancer Sci 109: 2697-2705, 2018.

9. Tomczak K, Czerwińska P and Wiznerowicz M: The cancer genome atlas (TCGA): An immeasurable source of knowledge. Contemp Oncol (Pozn) 19: A68-77, 2015.

10. Tang Z, Li C, Kang B, Gao G, Li C and Zhang Z: GEPIA: A web server for cancer and normal gene expression profiling and interactive analyses. Nucleic Acids Res 45: W98-W102, 2017.

11. Suzuki A, Horie T and Numabe Y: Investigation of molecular biomarker candidates for diagnosis and prognosis of chronic periodontitis by bioinformatics analysis of pooled microarray gene expression datasets in gene expression omnibus (GEO). BMC Oral Health 19: 52, 2019.

12. Grzmil M, Morin P Jr, Lino MM, Merlo A, Frank S, Wang Y, Moncayo G and Hemmings BA: MAP kinase-interacting kinase 1 regulates SMAD2-dependent TGF- $\beta$ signaling pathway in human glioblastoma. Cancer Res 71: 2392-2402, 2011.

13. Griesinger AM, Birks DK, Donson AM, Amani V, Hoffman LM, Waziri A, Wang M, Handler MH and Foreman NK: Characterization of distinct immunophenotypes across pediatric brain tumor types. J Immunol 191: 4880-4888, 2013.

14. Uhlen M, Zhang C, Lee S, Sjöstedt E, Fagerberg L, Bidkhori G, Benfeitas R, Arif M, Liu Z and Edfors F: A pathology atlas of the human cancer transcriptome. Science 357: eaan2507, 2017.

15. Livak KJ and Schmittgen TD: Analysis of relative gene expression data using real-time quantitative PCR and the 2(-Delta Delta C(T)) method. Methods 25: 402-408, 2001

16. Subramanian A, Tamayo P, Mootha VK, Mukherjee S, Ebert BL, Gillette MA, Paulovich A, Pomeroy SL, Golub TR, Lander ES and Mesirov JP: Gene set enrichment analysis: A knowledge-based approach for interpreting genome-wide expression profiles. Proc Natl Acad Sci USA 102: 15545-15550, 2005.

17. Mootha VK, Lindgren CM, Eriksson KF, Subramanian A, Sihag S, Lehar J, Puigserver P, Carlsson E, Ridderstråle M, Laurila E, et al: PGC-lalpha-responsive genes involved in oxidative phosphorylation are coordinately downregulated in human diabetes. Nat Genet 34: 267-273, 2003.
18. Wang G, Bie F, Li G, Shi J, Zeng Y and Du J: Study of the co-expression gene modules of non-small cell lung cancer metastases. Cancer Biomark: Dec 3, 2020 (Epub ahead of print). doi: 10.3233/CBM-201605.

19. Li X, Liu Z, Mi M, Zhang C, Xiao Y, Liu X, Wu G and Zhang L: Identification of hub genes and key pathways associated with angioimmunoblastic T-cell lymphoma using weighted gene co-expression network analysis. Cancer Manag Res 11: 5209-5220, 2019.

20. Chan BKC: Data analysis using R programming. Adv Exp Med Biol 1082: 47-122, 2018.

21. van Dam S, Vosa U, van der Graaf A, Franke L and de Magalhães JP: Gene co-expression analysis for functional classification and gene-disease predictions. Brief Bioinform 19: 575-592, 2018.

22. Schwartzbaum JA, Fisher JL, Aldape KD and Wrensch M: Epidemiology and molecular pathology of glioma. Nat Clin Pract Neurol 2: 494-503, 2006

23. Komori T: The 2016 WHO classification of tumours of the central nervous system: The major points of revision. Neurol Med Chir (Tokyo) 57: 301-311, 2017.

24. Chen R, Smith-Cohn M, Cohen AL and Colman H: Glioma subclassifications and their clinical significance. Neurotherapeutics 14: 284-297, 2017.

25. Apte RS, Chen DS and Ferrara N: VEGF in signaling and disease: Beyond discovery and development. Cell 176: 1248-1264, 2019.

26. Yunker CK, Golembieski W, Lemke N, Schultz CR, Cazacu S, Brodie C and Rempel SA: SPARC-Induced increase in glioma matrix and decrease in vascularity are associated with reduced VEGF expression and secretion. Int J Cancer 122: 2735-2743, 2008.

27. Tu Y, Zhong Y, Fu J, Cao Y, Fu G, Tian X and Wang B: Activation of JAK/STAT signal pathway predicts poor prognosis of patients with gliomas. Med Oncol 28: 15-23, 2011.

28. Zhang P, Chen FZ, Jia QB and Hu DF: Upregulation of microRNA-133a and downregulation of connective tissue growth factor suppress cell proliferation, migration, and invasion in human glioma through the JAK/STAT signaling pathway. IUBMB Life 71: 1857-1875, 2019.

29. Kumar DM,Patil V,Ramachandran B, Nila MV,Dharmalingam K and Somasundaram K: Temozolomide-Modulated glioma proteome: Role of interleukin-1 receptor-associated kinase-4 (IRAK4) in chemosensitivity. Proteomics 13: 2113-2124, 2013.

30. Sloan KE, Stewart JK, Treloar AF, Matthews RT and Jay DG: CD155/PVR enhances glioma cell dispersal by regulating adhesion signaling and focal adhesion dynamics. Cancer Res 65: 10930-10937, 2005.

31. Zheng Y, Miu Y, Yang X, Yang X and Zhu M: CCR7 Mediates TGF-beta1-induced human malignant glioma invasion, migration, and epithelial-mesenchymal transition by activating MMP2/9 through the nuclear factor KappaB signaling pathway. DNA Cell Biol 36: 853-861, 2017.

32. Luo J, Solimini NL and Elledge SJ: Principles of cancer therapy: Oncogene and non-oncogene addiction. Cell 136: 823-837, 2009.

33. Takada K, Kohashi K, Shimokawa M, Haro A, Osoegawa A Tagawa T, Seto T, Oda Y and Maehara Y: Co-Expression of IDO1 and PD-L1 in lung squamous cell carcinoma: Potential targets of novel combination therapy. Lung Cancer 128: 26-32, 2019.

34. Guan X, Zhang C, Zhao J, Sun G, Song Q and Jia W: CMTM6 overexpression is associated with molecular and clinical characteristics of malignancy and predicts poor prognosis in gliomas. EBioMedicine 35: 233-243, 2018.

This work is licensed under a Creative Commons Attribution-NonCommercial-NoDerivatives 4.0 International (CC BY-NC-ND 4.0) License. 